\title{
Erratum to: Geophysical estimation of permeability in sedimentary media with porosities from 0 to $50 \%$
}

\author{
Jesús Díaz-Curiel ${ }^{1, *}$, Bárbara Biosca ${ }^{2}$, and María Jesús Miguel ${ }^{3}$ \\ ${ }^{1}$ Department of Geological and Mining Engineering, Universidad Politécnica de Madrid, C/Ríos Rosas 21, 28003 Madrid, Spain \\ ${ }^{2}$ Department of Energy and Fuels, Universidad Politécnica de Madrid, C/Ríos Rosas, 21, 28003 Madrid, Spain \\ ${ }^{3}$ Spanish Ministry of Science and Innovation, Paseo de la Castellana 162, 28046 Madrid, Spain
}

\section{Accepted: 14 April 2021}

Erratum for: Oil \& Gas Science and Technology - Rev. IFP Energies nouvelles 71, 27 (2016) - https://doi.org/10.2516/ogst/2014053

Regarding the article [Díaz-Curiel, J., Biosca, B., \& Miguel, M.J. (2016) Geophysical estimation of permeability in sedimentary media with Porosities from 0 to $50 \%$. Oil \& Gas Science and Technology-Revue d'IFP Energies nouvelles, 71(2), 27.], published March 19th, 2015, an error has occurred in the version of article OGST-2014053 in the coefficients $\mathrm{f}_{2}$ and $\mathrm{f}_{3}$ of Equation (4a), the ratio $k(F)$, whose values are reflected in Equation (4b) and Figure 3. This is the fundamental equation of the study, on which the rest of the relationships are developed, so the propagation of this error is reflected in other expressions and graphs, whose modifications are detailed. Consequently, some of the values used in Validation (Figs. 5 and 6) change, without significantly changing the final result of the Validation. Some of the values of the Examples explaining the behaviour of the equation $k(\varnothing)(5 \mathrm{~b})$ also change, which in this case makes it necessary to change the results of the examples analysed.

Figure 3 should be:

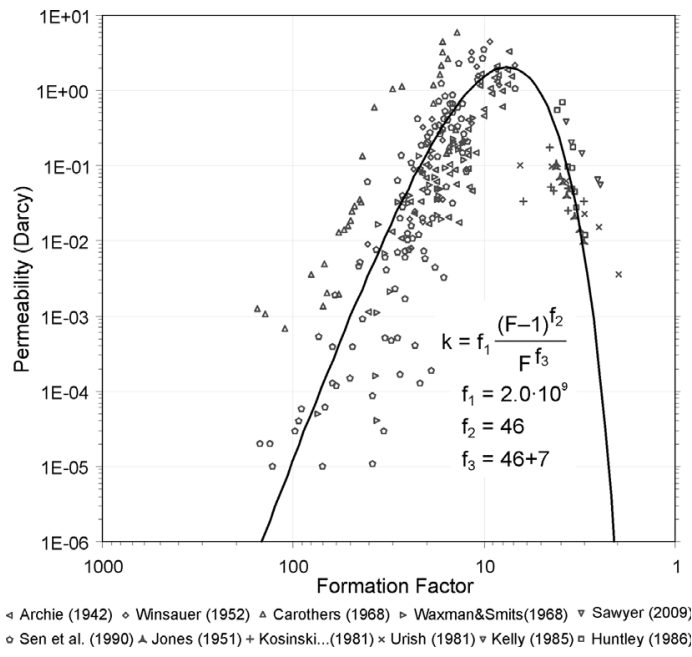

\footnotetext{
* Corresponding author: j.diazcuriel@upm.es
}

instead of:

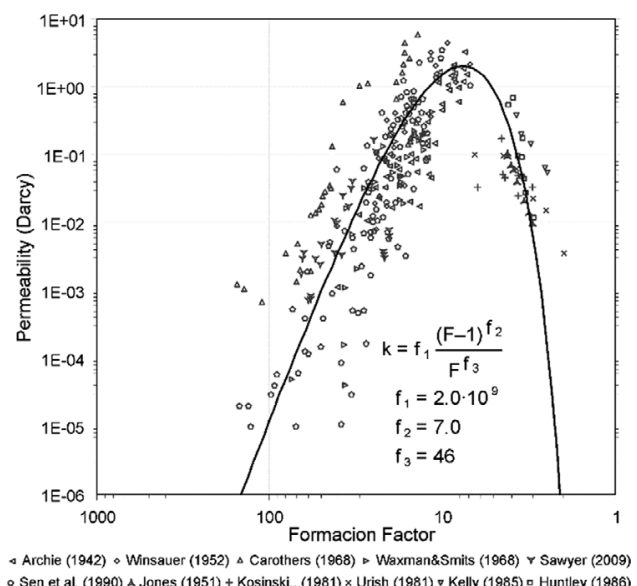

Equation (4b) should be:

instead of:

$$
k=2.0 \cdot 10^{9} \frac{(F-1)^{46}}{F^{46+7}}
$$

$$
k=2.0 \cdot 10^{9} \frac{(F-1)^{7}}{F^{46}}
$$

Figure 4 should be:

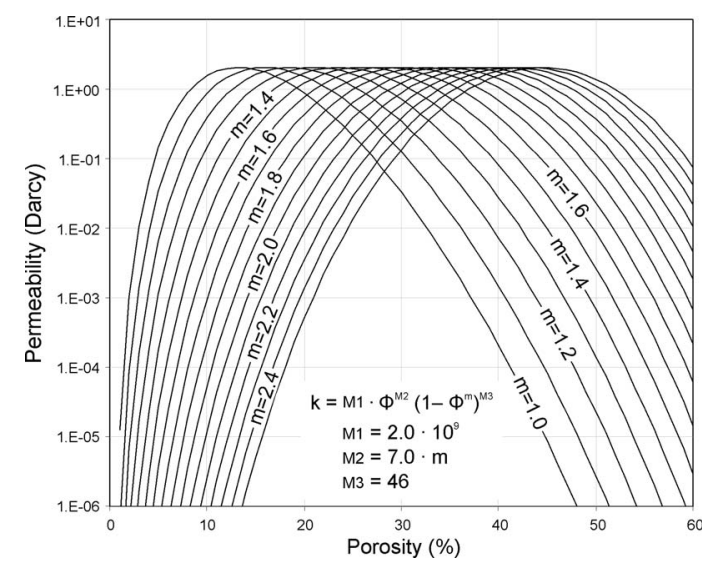


instead of:

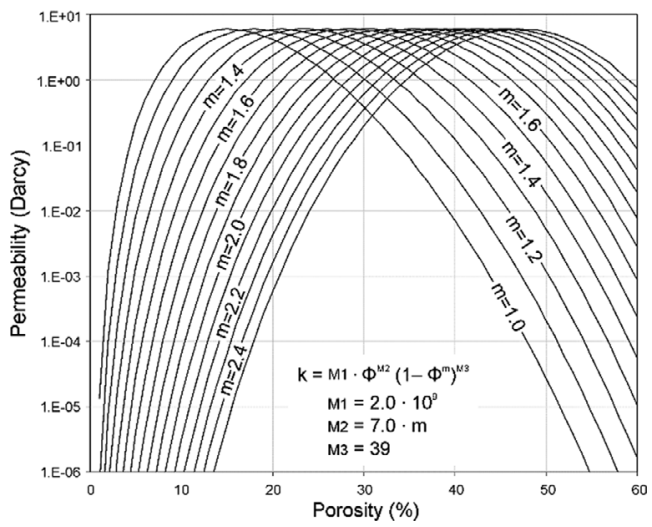

Equation (5b) should be:

$$
k=2.0 \cdot 10^{9} \cdot \emptyset^{7 m} \cdot(1-\emptyset)^{46}
$$

instead of:

$$
k=2.0 \cdot 10^{9} \cdot \emptyset^{7 m} \cdot(1-\emptyset)^{39}
$$

Figure 5 should be:

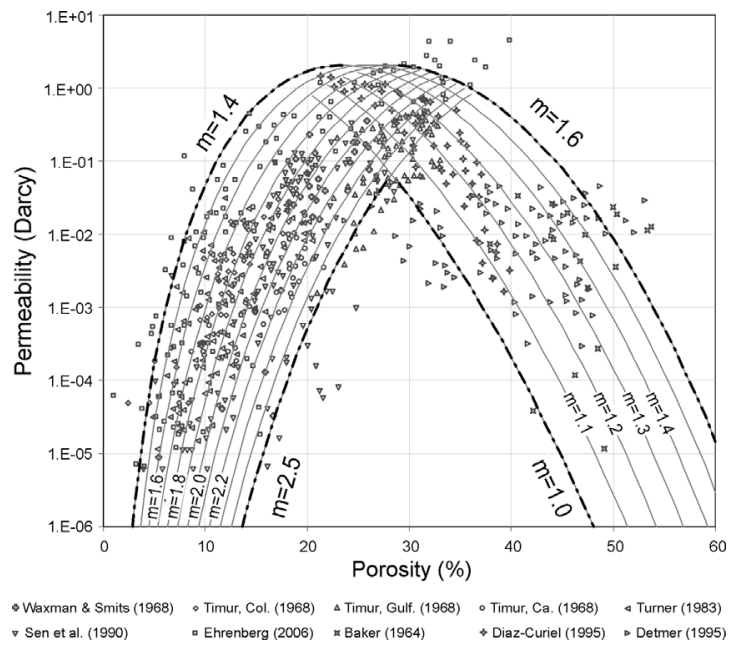

instead of:

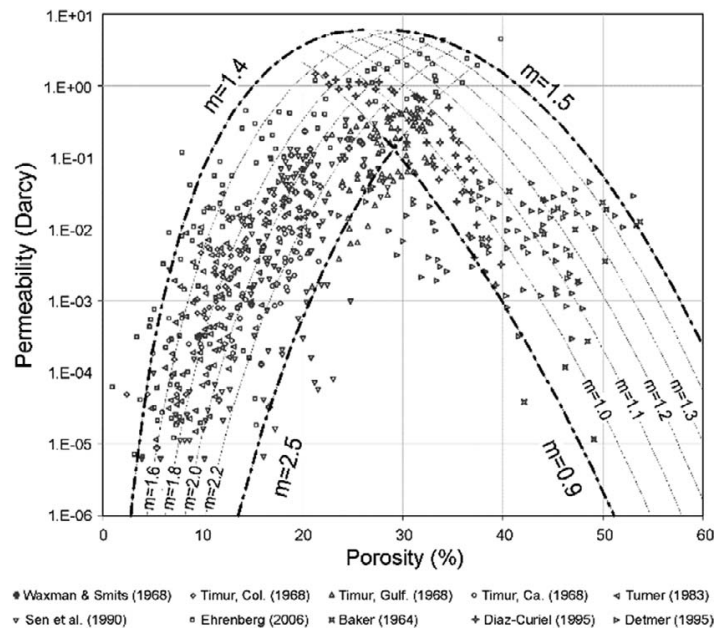

On pages, 10 and 11 the values of the examples must be corrected.

Page 10, it should be read:

As it is observed in Figure 5, data from these studies are included in the range of curves obtained from Equation (5b) of $k(\varnothing)$, and most of the data set match a particular curve, that is to say, with a certain cementation exponent. The cementation exponent values vary, for the set of those data, between $\mathbf{1 . 4 0}$ and 2.50, and these are quite reasonable if we consider that clay content has not been taken into account. The cementation exponent of the $k(\varnothing)$ expression that embraces the Waxman and Smits (1968) data is between $\mathbf{1 . 6 0}$ and $\mathbf{2 . 4 5}$, for the $99 \%$ of the data from Turner (1983) is between $\mathbf{1 . 3 0}$ and $\mathbf{2 . 1 0}$, and for the $96 \%$ of Timur (1968) data from Colorado, is between $\mathbf{1 . 4 5}$ and $\mathbf{1 . 9 5}$.

instead of:

As it is observed in Figure 5, data from these studies are included in the range of curves obtained from Equation (5b) of $k(\varnothing)$, and most of the data set match a particular curve, that is to say, with a certain cementation exponent. The cementation exponent values vary, for the set of those data, between 1.35 and 2.50, and these are quite reasonable if we consider that clay contefnt has not been taken into account. The cementation exponent of the $k(\varnothing)$ expression that embraces the Waxman and Smits (1968) data is between 1.9 and 2.5 , for the $99 \%$ of the data from Turner (1983) is between 1.5 and 2.2, and for the $96 \%$ of Timur (1968) data from Colorado, is between 1.6 and 2.1 .

Page 11, it should be read:

We have represented the curves given by Equation (5b) for $K(\varnothing)$, for values of $m=\mathbf{1 . 0 0}$ and $m=\mathbf{1 . 6 0}$, leaving out of range data from the upper right, since they have permeability values of $\sim 0.1$ Darcy for porosities of about $50 \%$, which are fairly uncommon simultaneously. The range of " $m$ " values for Equation (5b), of $k(\varnothing)$, that set the limits for all Díaz-Curiel (1995) data are between $\mathbf{1 . 0 5}$ and 1.45, for the $90 \%$ of Baker et al. (1964) data between $\mathbf{0 . 9 5}$ and 1.45, and for the $93 \%$ of Detmer (1995) data between $\mathbf{0 . 9 5}$ and $\mathbf{1 . 6 0}$.

instead of:

We have represented the curves given by Equation (5b) for $K(\varnothing)$, for values of $m=0.9$ and $m=1.5$, leaving out of range data from the upper right, since they have permeability values of $\sim 0.1$ Darcy for porosities of about $50 \%$, which are fairly uncommon simultaneously. The range of " $m$ " values for Equation (5b), of $k(\varnothing)$, that set the limits for all Díaz-Curiel (1995) data are between 0.9 and 1.20, for the $90 \%$ of Baker et al. (1964) data between 0.95 and 1.5, and for the $93 \%$ of Detmer (1995) data between 0.9 and 1.4. 
Page 11, it should be read:

In order to know a degree of uncertainty for Equation (5b) of $k(\varnothing)$, we have taken the $(k, \varnothing)$ values from Glover and Walker (2009). The mean difference with the $k(\varnothing)$ values obtained from Equation $(5 \mathrm{~b})$ for $m=1.90$, is $\mathbf{0 . 2}$ order of magnitude, which once again we consider a sufficiently close approximation for the goal of this work. We have showed both together in Figure 6, in which it can be seen that the cementation exponent from Glover and Walter (2009) data for porosities $\geq 19 \%$ are smaller than the remainder

instead of:

In order to know a degree of uncertainty for Equation (5b) of $k(\varnothing)$, we have taken the $(k, \varnothing)$ values from Glover and Walker (2009). The mean difference with the $k(\varnothing)$ values obtained from Equation (5b) for $m=1.9$, is 0.3 order of magnitude, which once again we consider a sufficiently close approximation for the goal of this work. We have showed both together in Figure 6 , in which it can be seen that the cementation exponent from Glover and Walter (2009) data for porosities $\geq 19 \%$ are smaller than the remainder.

Page 11, it should be read:

We performed the same test for unconsolidated media on extracted data from the work of Díaz-Curiel (1995) and contrasted them with the $k(\varnothing)$ values obtained from Equation (5b) for $m=\mathbf{1 . 2 2}$ (Fig. 6), resulting in an uncertainty average of $\mathbf{0 . 2}$ order of magnitude. In this case, the highest cementation exponent takes place for porosities around $\mathbf{2 2 - 2 8 \%}$.

instead of:

We performed the same test for unconsolidated media on extracted data from the work of Díaz-Curiel (1995) and contrasted them with the $k(\varnothing)$ values obtained from Equation (5b) for $m=1.0$ (Fig. 6), resulting in an uncertainty average of 0.31 order of magnitude. In this case, the highest cementation exponent takes place for porosities around $27-35 \%$.

Figure 6 should be:

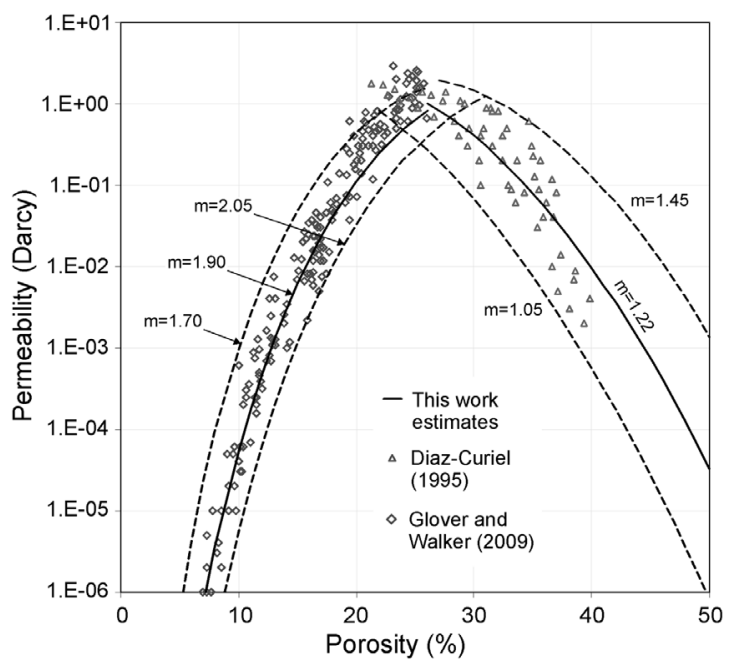

instead of:

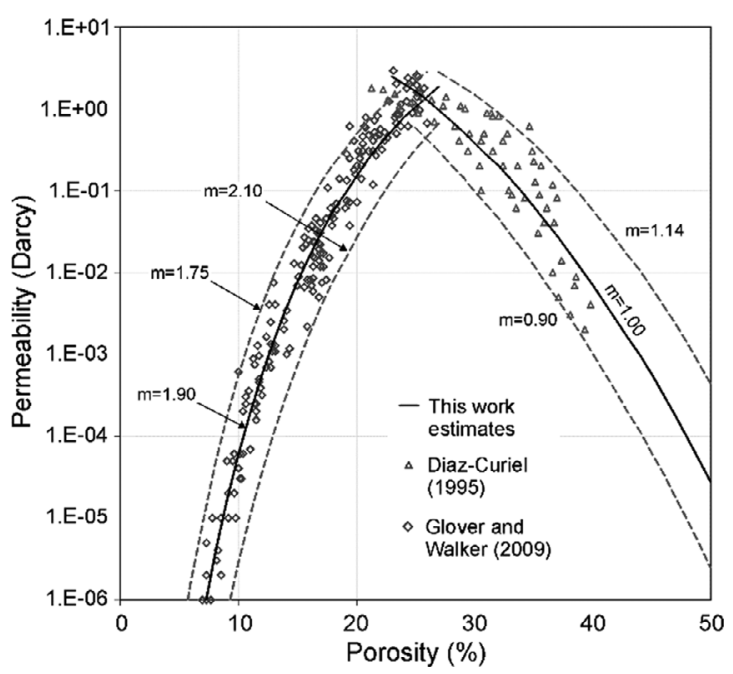

On pages, 14 and 15 the values of the examples must be corrected.

Page 14, it should be read:

Consider, first, two media with the same permeability, for example, a sandstone with $\varnothing=\mathbf{5 . 8} \%$ and $m=1.40$, and another with $\varnothing=15 \%$ and $m=\mathbf{2 . 0 0}$; the formation factors are close to $F=\mathbf{5 3 . 7}$ for both media. The most probable reason why these media have the same permeability (6.4. $10^{-4}$ Darcy) lies in the fact that the latter is more cemented. Similarly, an unconsolidated sand with $\varnothing=\mathbf{3 9} \%$ and $m=\mathbf{1 . 4 0}$ has a formation factor of $\boldsymbol{F}=\mathbf{3 . 7 4}$, which is practically equal to that of a silt with $\varnothing=46 \%$ and $m=\mathbf{1 . 7 0}$, and both have the same permeability value $\left(\mathbf{1 . 2} \cdot 10^{-1}\right.$ Darcy). In this case, the most probable reason why these media have the same permeability, despite the difference of grain size, is that they present very different sorting.

instead of:

Consider, first, two media with the same permeability, for example, a sandstone with $\varnothing=6 \%$ and $m=1.40$, and another with $\varnothing=15 \%$ and $m=$ 2.10; the formation factors are close to $F=51.3$ for both media. The most probable reason why these media have the same permeability $\left(9.7 \cdot 10^{-4}\right.$ Darcy) lies in the fact that the latter is more cemented. Similarly, an unconsolidated sand with $\varnothing=38 \%$ and $m=1.20$, has a formation factor of $F=3.19$, which is practically equal to that of a silt with $\varnothing=46 \%$ and $m=1.50$, and both have the same permeability value $\left(2.5 \cdot 10^{-1}\right.$ Darcy). In this case, the most probable reason why these media have the same permeability, despite the difference of grain size, is that they present very different sorting.

Page 15, it should be read:

On the other hand, if we consider different media with the same porosity value, it is noticeable that a 
high-permeability sandstone, for example with $\varnothing=11 \%$ and $m=1.60$, has much greater permeability $\left(\mathbf{9 . 2} \cdot \mathbf{1 0}^{-\mathbf{3}}\right.$ Darcy) than a more cemented sandstone with $\varnothing=11 \%$ and $m=2.00$ (permeability 4.2 $\cdot 10^{-5}$ Darcy). However, unconsolidated sands with $\varnothing=43 \%$ and $m=\mathbf{1 . 8 0}$ have more permeability $\left(\mathbf{5 . 5} \cdot \mathbf{1 0}^{-\mathbf{1}}\right.$ Darcy) than sandy silts with $\varnothing=43 \%$ and $m=\mathbf{1 . 3 0}$ (permeability $\mathbf{7 . 0} \cdot \mathbf{1 0}^{-\mathbf{3}}$ Darcy). Therefore, for a given porosity in consolidated media, the higher the " $m$ " values, which corresponds to higher formation factor, the lower the permeability values. On the contrary, the opposite applies to unconsolidated media.

instead of:

On the other hand, if we consider different media with the same porosity value, it is noticeable that a highpermeability sandstone, for example with $\varnothing=11 \%$ and $m=1.60$, has much greater permeability $\left(1.2 \cdot 10^{-2}\right.$ Darcy) than a more cemented sandstone with $\varnothing=11 \%$ and $m=2.00$ (permeability $8.3 \cdot 10^{-5}$ Darcy). However, unconsolidated sands with $\varnothing=43 \%$ and $m=1.60$ have more permeability (1.3 Darcy) than sandy silts with $\varnothing=43 \%$ and $m=1.20$ (permeability $3.7 \cdot 10^{-2}$ Darcy). Therefore, for a given porosity in consolidated media, the higher the " $m$ " values, which corresponds to higher formation factor, the lower the permeability values. On the contrary, the opposite applies to unconsolidated media.

Figure 10 should be:

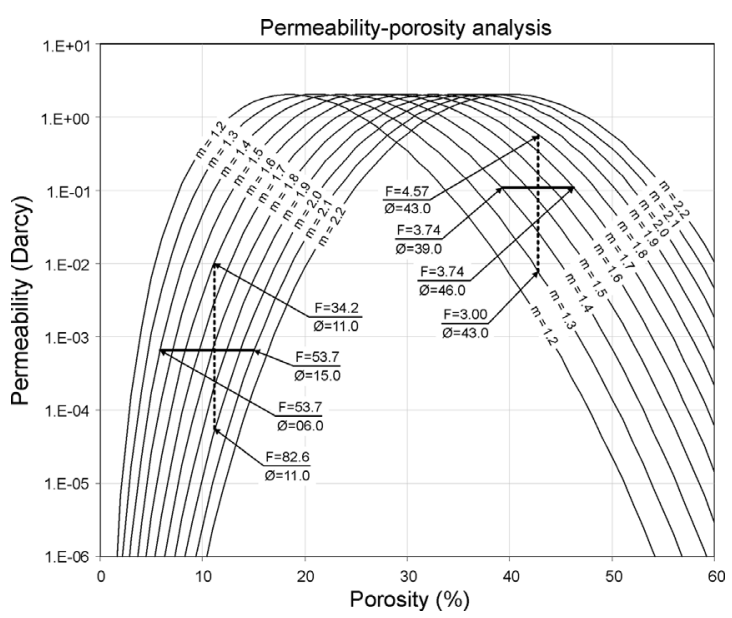

instead of:

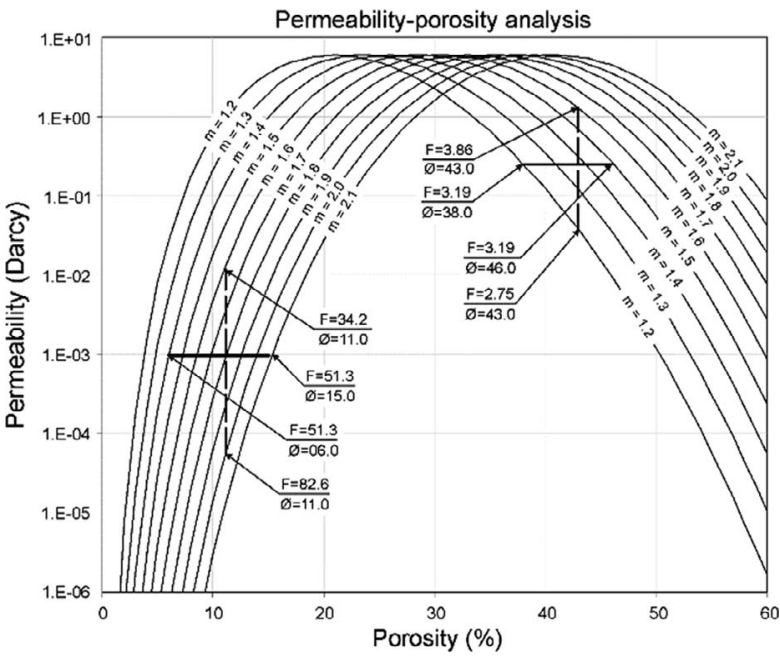

In Conclusion, the $\mathbf{2}^{\text {nd }}$ equation should be (This is the previously corrected Eq. (4b)):

$$
k=2.0 \cdot 10^{9} \frac{(F-1)^{46}}{F^{46+7}}
$$

instead of:

$$
k=2.0 \cdot 10^{9} \frac{(F-1)^{7}}{F^{46}}
$$

The $\mathbf{3}^{\text {rd }}$ equation should be (This is the previously corrected Eq. (5b)):

$$
k=2.0 \cdot 10^{9} \cdot \emptyset^{7 m} \cdot(1-\emptyset)^{46}
$$

instead of:

$$
k=2.0 \cdot 10^{9} \cdot \emptyset^{7 m} \cdot(1-\emptyset)^{39}
$$

